\title{
PERFORMANCE OF THE SPS BEAM SPECTROMETER CHAMBERS UNDER VERY HIGH FLUXES
}

\author{
A. PLACCI, M. RABANY and G. VISMARA \\ CERN, Geneva, Switzerland
}

\begin{abstract}
Five beam spectrometers each consisting of four $100 \times 100 \mathrm{~mm}^{2}, 1 \mathrm{~mm}$ wire spacing, multiwire proportional chambers, are operational in the secondary beams of the CERN SPS. To determine the ultimate performance of the chambers and associated electronics, capable of working at a threshold of $0.5 \mu \mathrm{A}$ and transmitting signals on $500 \mathrm{~m}$ twisted pair cables at rates of $25 \mathrm{MHz}$ /wire, we have studied the behaviour of the chambers under very high beam fluxes $\left(\sim 10^{7}\right.$ particles/s). Up to $10^{5}$ particles $/\left(\mathrm{mm}^{2} \cdot \mathrm{s}\right)$ efficiencies as high as $90 \%$ can be reached.
\end{abstract}

\section{Introduction}

At the CERN SPS (Super-Proton-Synchrotron) all the hadronic secondary beams are provided with a momentum measuring facility in order to allow experiments to determine accurately the momentum of the incoming beam particles associated with their events. Each spectrometer consists of a set of four multiwire proportional chambers with $1 \mathrm{~mm}$ sense wire spacing, sufficient, in most of the cases, to obtain a momentum resolution $(\Delta p / p)$ of one pion mass. In order to withstand the high beam fluxes [from $5 \times 10^{5}$ to $10^{7}$ particles $/ \mathrm{s}$, leading to densities up to a few $10^{4}$ particles $\left./\left(\mathrm{mm}^{2} \cdot \mathrm{s}\right)\right]$ a very fast electronic chain and readout system') has been specially implemented.

This paper reports on a detailed investigation into the ultimate performance of one chamber and its associated electronics. The efficiency of the chamber was measured as a function of beam density for two inorganic gas mixtures $\mathrm{A}(50 \%)+$ $\mathrm{CO}_{2}(50 \%)$ and $\mathrm{A}(50 \%)+\mathrm{CO}_{2}(49,7 \%)+$ Freon 13 $\mathrm{B} 1(0.3 \%)$. A special attention was devoted to sep- arate the contribution to the system inefficiency coming from the electronics and the space charge effects in the chamber. Also the chamber time jitter was measured in order to have the minimum resolving time compatible with an acceptable efficiency.

\section{Experimental method}

\subsection{THE SET-UP}

The measurements have been performed on one chamber of the $\mathrm{H} 3$ beam spectrometer in the SPS West Experimental Area. The beam was focussed in both planes a few meters before the chamber. The total beam flux and density were changed by varying the beam momentum and foci position. Furthermore, in order to change the counting rate/wire (by a factor 2), while keeping the beam density constant, we could collimate part of the beam.

The physical set-up is shown in fig. 1. Two scintillators, each having a $3 \times 3 \mathrm{~mm}^{2}$ cross-section and a thickness of $4 \mathrm{~mm}$, were used to define the

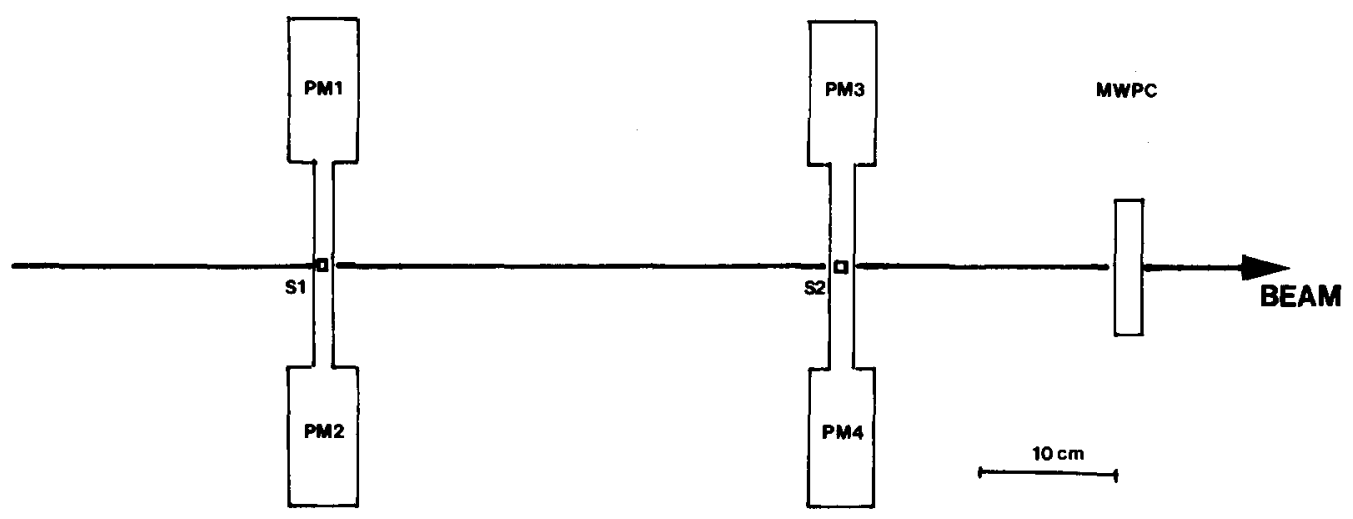

Fig. 1. Schematic vertical cross-section of the set-up. 
beam crossing the chamber. To reduce spurious triggers due to the beam halo (mainly on the horizontal bending plane), each scintillator was viewed, through an air light guide, by two photomultipliers positioned on the vertical axis. For practical reasons the two scintillators $\left(S_{1}, S_{2}\right)$ have been mounted in front of the chamber, on the same scanning table (XXYT). The maximum displacement (computer controlled) was $\pm 50 \mathrm{~mm}$ on both axes, with an accuracy better than $\pm 0.05 \mathrm{~mm}$. Fig. 2 shows a typical beam profile together with the cross-section of the beam defining scintillators.

The main parameters of the chamber, which is
TABLE 1

Parameters of the multiwire proportional chamber.

Sensitive area

Sense wire spacing

$100 \times 100 \mathrm{~mm}^{2}$

Sense wire diameter (gold-plated tungsten)

Spacing between high voltage and signal plane

$1 \mathrm{~mm}$

$10 \mu \mathrm{m}^{\mathrm{a}}$

High voltage plane - aluminium foil thickness

$5 \mathrm{~mm}$

$10 \mu \mathrm{m}$

a On both edges of the sense plane are included three addi. tional guard wires of $30,50,100 \mu \mathrm{m}$ diameter respectively.

of a CERN standard design, are listed in table 1.

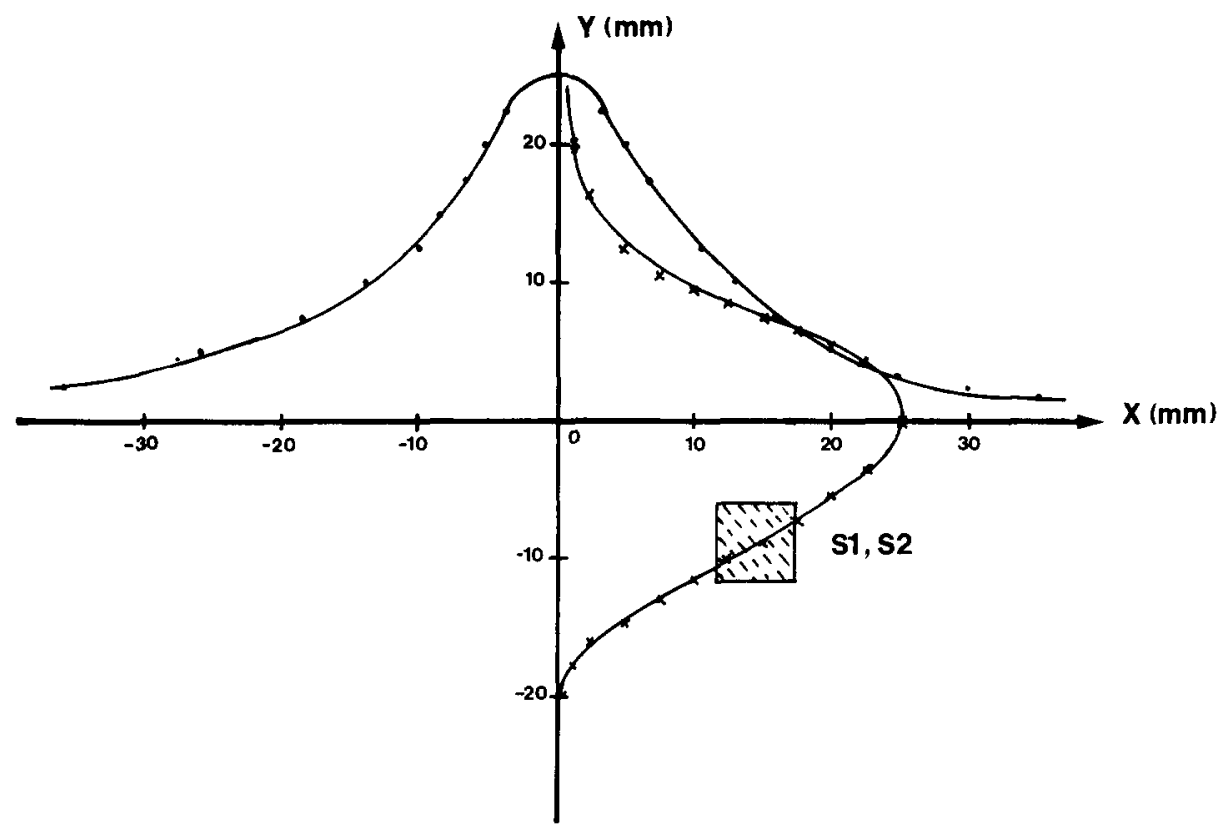

Fig. 2. Horizontal and vertical beam profile measured by the scintillators.

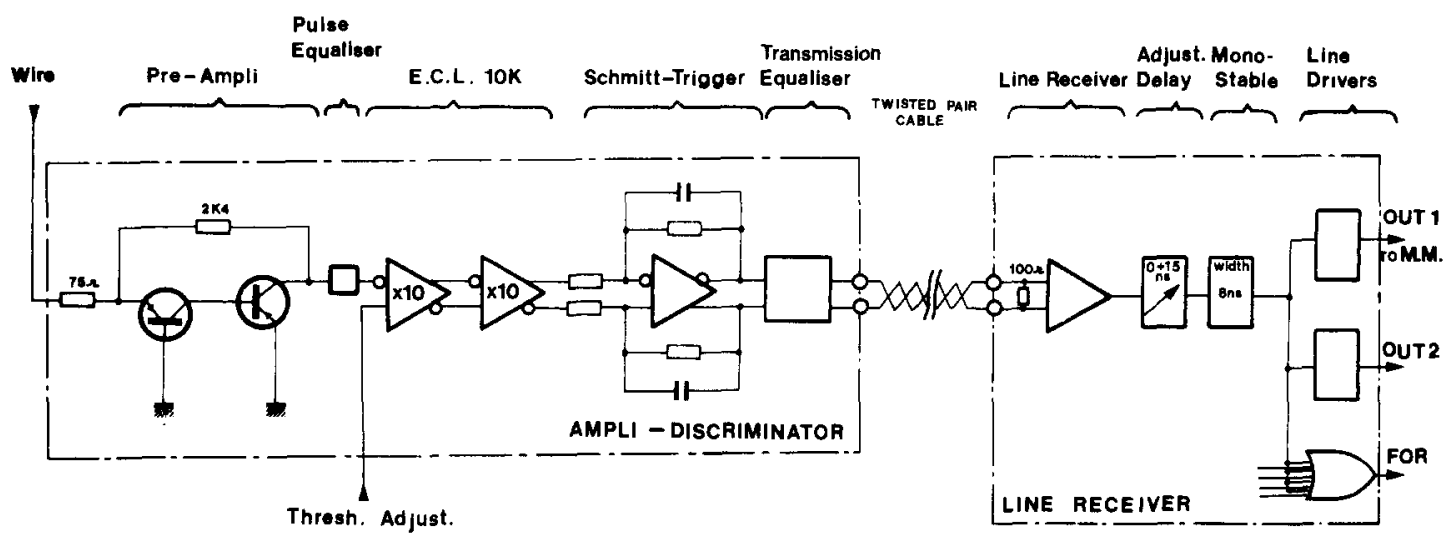

Fig. 3. Block diagram of the chamber electronics. 


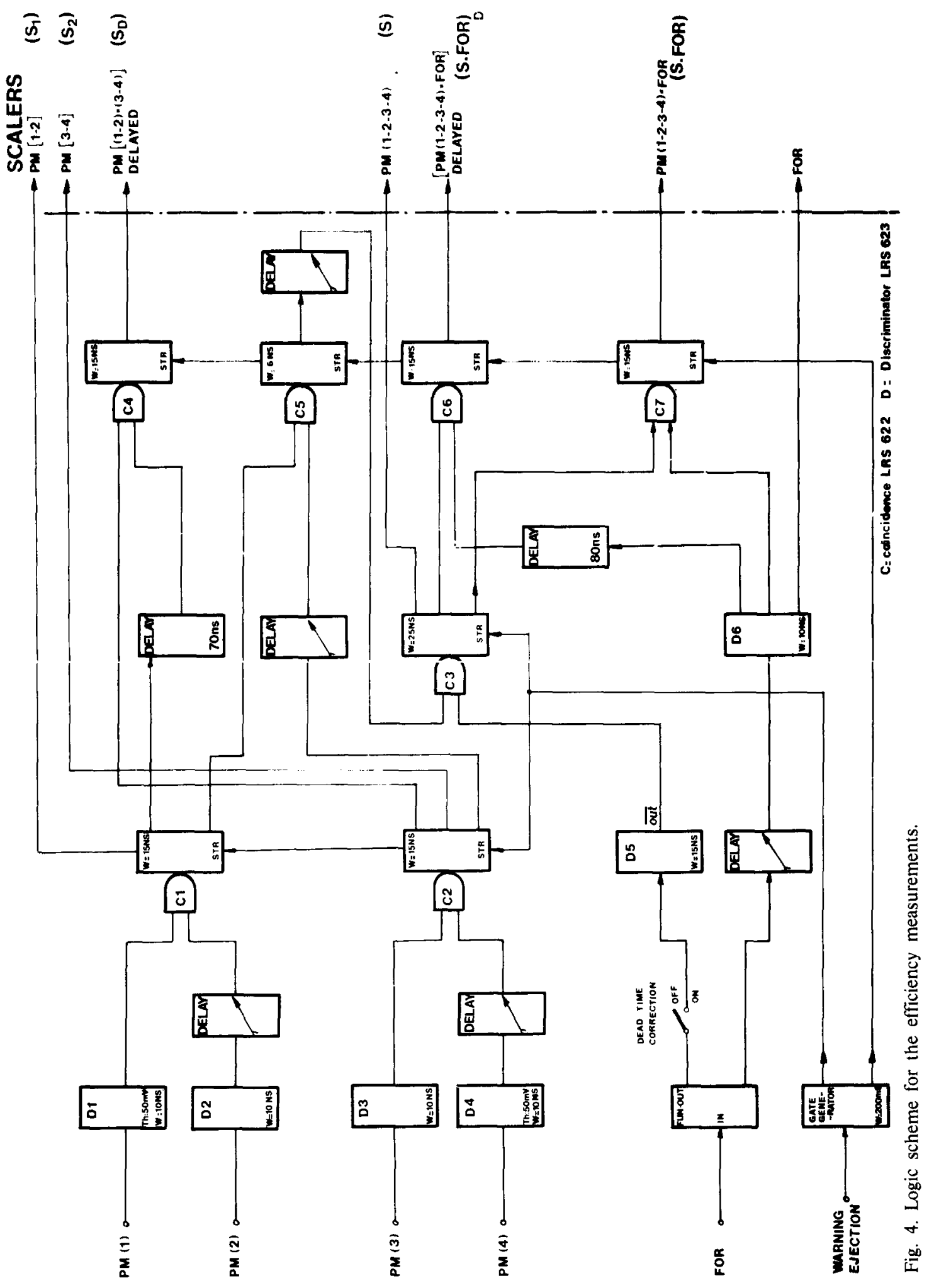




\subsection{The ELECTRONICS}

The signals from the chamber sense wires were fed into a current amplifier-discriminator stage ${ }^{2}$ ), the main parameters of which are listed in table 2; the logic outputs were sent through $240 \mathrm{~m}$ (1200 ns) of twisted pair cables to the counting room and reformed by a Line Receiver (fig. 3). The rather long transmission and a final timing adjustment (within $\pm 1 \mathrm{~ns}$ ) among the various channels, through an active delay, increased the double pulse resolution time to 38 ns. For our test, in front of the Line Receiver, which normally performs the OR function of 16 wires, a switching unit allowed to select the OR of a smaller group of wires. The complete electronics logic scheme is shown in fig. 4.

\subsection{SPill time measurement}

Since the effective beam spill time $\left(T_{\text {eff }}\right)$ had large fluctuations from burst to burst, due to the presence of time structure in the SPS slow ejection (1.4 $\mathrm{s}$ flat-top), a measurement of $T_{\text {eff }}$ was needed for normalisation purposes. The method used is based on the measurements of the single rates (which are already a coincidence between two photomultipliers) and delayed coincidence between the triggers $\left(S_{1}, S_{2}\right.$ and $S_{D}$ of fig. 4). $T_{\text {eff }}$ is then given by

$T_{\text {eff }}=\tau S_{1} S_{2} / S_{\mathrm{D}}$,

where $\tau$ is the coincidence overlapping time (30 ns).

Furthermore, to allow the chamber to reach a

TABLE 2

Parameters of the amplifier-discriminator.

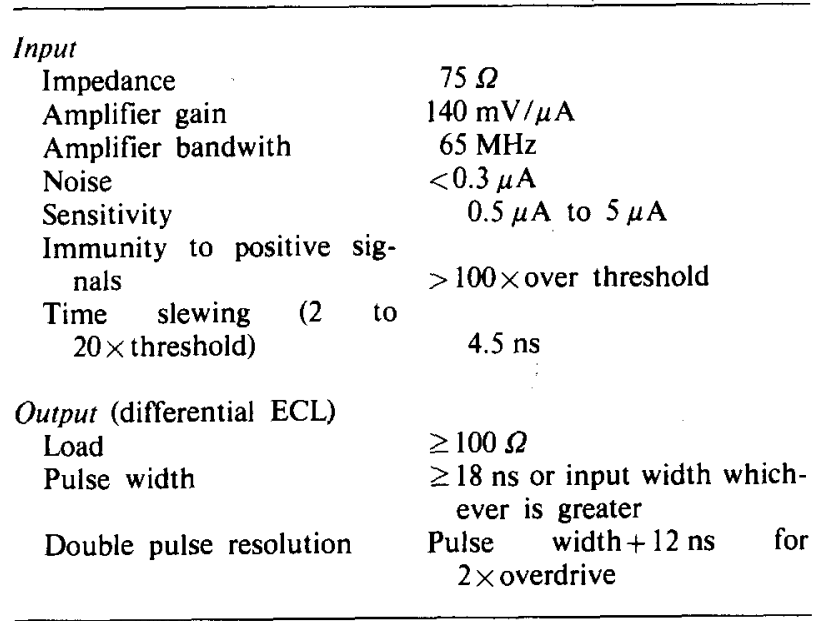

steady state due to the building-up of the space charge, the measurements were gated-off during the first $200 \mathrm{~ms}$ of the ejection.

\subsection{BEAM DENSITY AND EFFICIENCY DEFINITION}

To define the beam density impinging on the chamber, the coincidence between $S_{1}$ and $S_{2}$ was not used, because of the large beam divergence which could introduce errors as big as $30 \%$ for a $10 \mathrm{~mm}$ lateral displacement of the two scintillators. We then relied on the two counting rates of $\mathrm{S}_{1}$ and $\mathrm{S}_{2}{ }^{*}$ which were differing by less than $2 \%$, when the triggers were positioned on the beam axis. Therefore the beam density $D$ was defined:

$D=\frac{1}{2}\left(S_{1}+S_{2}\right) \frac{1}{T_{\text {eff }} A}$ particles $/\left(\mathrm{mm}^{2} \cdot \mathrm{s}\right)$,

where $A=9 \mathrm{~mm}^{2}$ is the cross-section of two scintillators. Dimensions and alignment of the triggers have been carefully measured so as to contribute less than $5 \%$ to the error on $D$.

On the other hand, as normalisation for the efficiency measurement, we have taken the coincidence $(S)$ between scintillators, which being a four-fold coincidence, is highly insensitive to beam halo investing the photomultipliers. Furthermore, we made a careful check that a minimum of four wires covered completely the area of the scintillators.

The chamber time jitter (which includes the electronic time slewing) has been measured for a threshold of $0.5 \mu \mathrm{A}$ and the best results were obtained for a gas mixture of $\mathrm{A}+\mathrm{CO}_{2}+$ Freon

\footnotetext{
* Under conditions of maximum density the rate of $S_{1}$ and $S_{2}$ was always $\leq 7 \times 10^{5}$ counts $/ \mathrm{s}$, so that saturation effects were negligible.
}

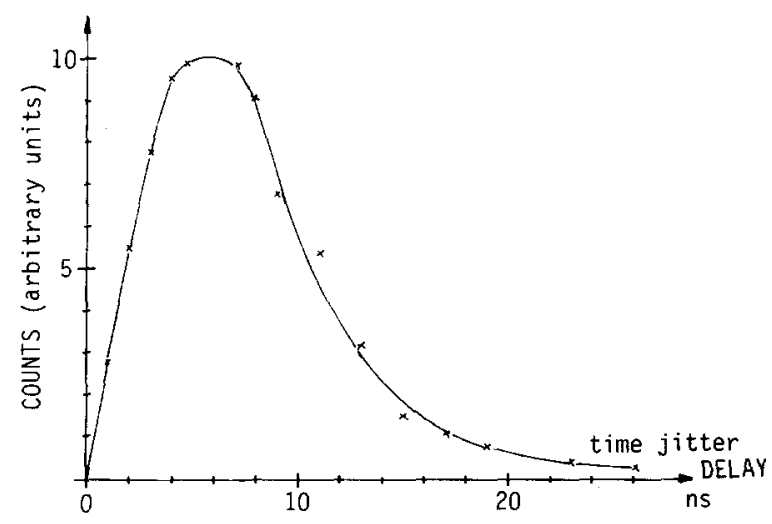

Fig. 5. Timing jitter of the chamber pulses. The curve is for a mixture of $\mathrm{A}(50 \%)+\mathrm{CO}_{2}(49.7 \%)+$ Freon $13 \mathrm{~B} \mathrm{1}(0.3 \%)$; the high voltage is $5 \mathrm{kV}$ and the discriminator threshold $0.5 \mu \mathrm{A}$. 
$(50 \%+49.7 \%+0.3 \%)$ (fig. 5). A strobe width as small as $20 \mathrm{~ns}$ could be used with an efficiency loss of about 1\%; however, to avoid timing adjustment, when changing the voltage on the chamber or the discriminator threshold, a $25 \mathrm{~ns}$ strobe pulse was used. In this way, setting a width of $10 \mathrm{~ns}$ for the sense wires OR (FOR), efficiencies as high as $99.4 \%$ could be obtained (fig. 6).

At high rates the calculation of the efficiency is affected by the random coincidences due to particles interacting on the selected wires but not on the scintillators. This error, which is a function of the intensity, also depends on the chamber efficiency, being negligible at high percentage values but quite important at lower ones. For this reason, we have measured the delayed coincidence between FOR and S (S. FOR) $)_{D}$ and corrected for it the chamber efficiency Eff which was then defined as

$$
\mathrm{Eff}=\frac{(\mathrm{S} . \mathrm{FOR})-(\mathrm{S} . \mathrm{FOR})_{\mathrm{D}}}{\mathrm{S}-(\mathrm{S} . \mathrm{FOR})_{\mathrm{D}}}
$$

An additional feature introduced in the measurement, was the possibility to gate-off all strobe pulses $\mathrm{S}$, coming after reception of a FOR signal during 60-80 ns, depending on the chamber pulse jitter (figs. 4 and 7). For this, the output of $C_{5}$ was made as short as possible (6 ns) and came $20 \mathrm{~ns}$ before the latest FOR pulse $\left(D_{5} \overline{\text { OUT }}\right)$, produced by a retriggerable monostable. This artificial deadtime on the strobe $S$ allowed to separate the two different contributions to the inefficiency of the system, coming from the electronics and from the space charge in the chamber.

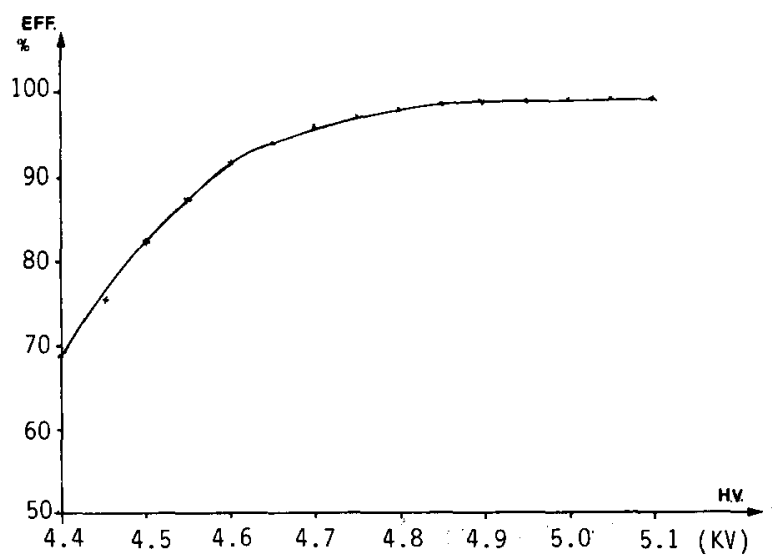

Fig. 6. High voltage plateau for a threshold $=0.5 \mu \mathrm{A}$ and a mixture of $\mathrm{A}(50 \%)+\mathrm{CO}_{2}(49.7 \%)+$ Freon $13 \mathrm{~B} 1(0.3 \%)$; the beam density is about $10^{3}$ particles $/\left(\mathrm{mm}^{2} \cdot \mathrm{s}\right)$.
The electronics contribution to the inefficiency is determined by the "dead time" and the "occupation time" or time during which the amplifier output is over the discriminator threshold; in both cases the inefficiency is proportional to the single wire rate.

In our system, the "dead time" resulted in a double pulse resolution of about $38 \mathrm{~ns}$ (a minimum width of $18 \mathrm{~ns}$ followed by a $20 \mathrm{~ns}$ dead time). Considering now the probability $P_{2}(t)^{*}$ that two events, randomly distributed, occur in a time interval $\Delta t$, the "dead time" can be easily converted in loss of efficiency at a given event rate.

On the other hand the "occupation time" is more difficult to evaluate because it depends on many parameters as wire capacitance, wire load resistance, gas mixture, chamber gain, etc.

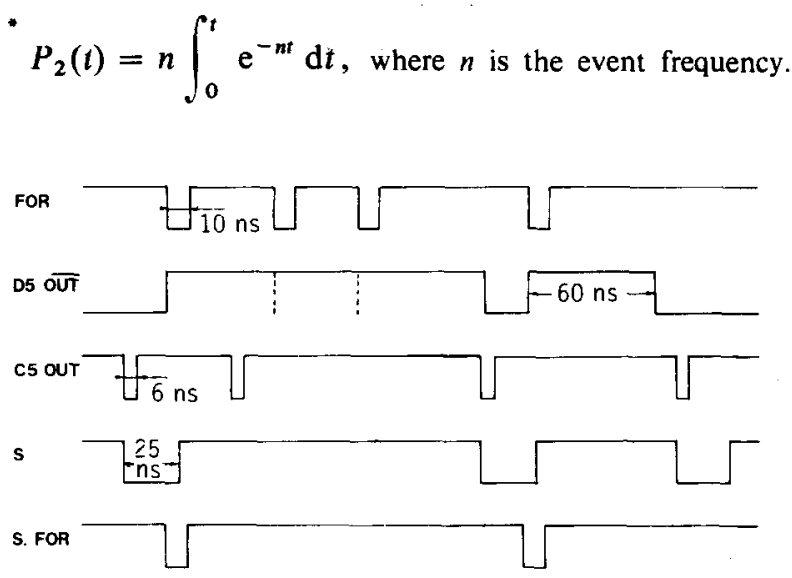

Fig. 7. Timing sequence of the electronic dead-time compensation. The figure shows the veto action of a chamber pulse (D5 $\overline{\text { OUT }}$ started by a FOR pulse) on the trigger coincidence (C5 OUT) generating the strobe $\mathrm{S}$.

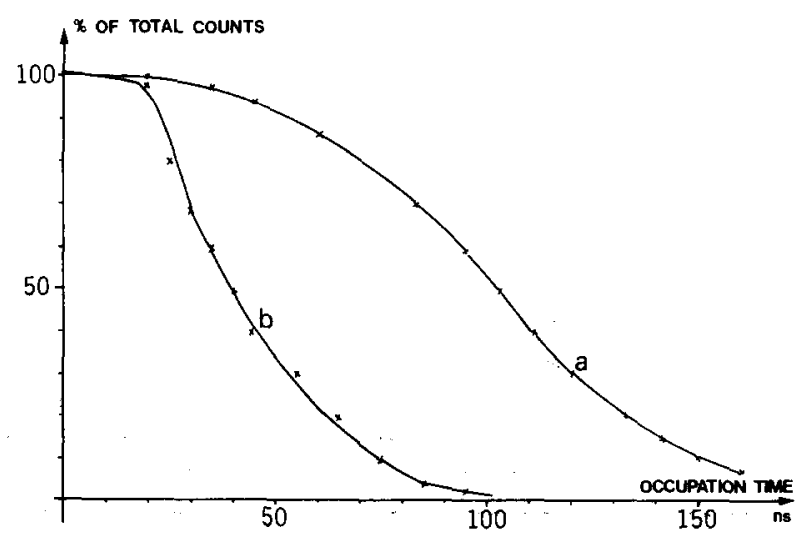

Fig. 8. Integral distribution of the "occupation time" without (curve a) and with (curve b) RC compensation. The curves are for a mixture of $\mathrm{A}(50 \%)+\mathrm{CO}_{2}(50 \%)$, a high voltage $=4.8 \mathrm{kV}$ and a threshold $=0.5 \mu \mathrm{A}$. 
Assuming that the chamber pulse current $i(t)$ can be represented by the expression ${ }^{3}$ )

$i(t)=i(0)\left(P \mathrm{e}^{-t / T_{1}}+Q \mathrm{e}^{-t / T_{2}}\right)$,

where, for our chamber and gas mixture, $P=0.8$, $Q=0.2, T_{1}=3 \mathrm{~ns}$ and $T_{2}=20 \mathrm{~ns}$, it is clear that, to obtain a short occupation time, a compensation of the longest time-constant term has to be introduced. This has been achieved in the preamplifier stage by using an RC equaliser, which has reduced the occupation time from a typical value of $105 \mathrm{~ns}$ to $45 \mathrm{~ns}$ (fig. 8 ).

\section{Results and data interpretation}

The contribution to the inefficiency of the system due to the electronics is clearly shown in fig. 9 which represents some measured inefficiencies with and without dead time correction, as a function of the FOR intensity under conditions of low beam density $\left[10^{3}\right.$ particles $\left./\left(\mathrm{mm}^{2} \cdot \mathrm{s}\right)\right]$. The slope difference of the uncompensated curves is determined by the different contribution of the occupation time following the chamber gain. For example, considering the probability $P_{2}(t)$, one deduces an inefficiency of about $2 \%$ at a rate of

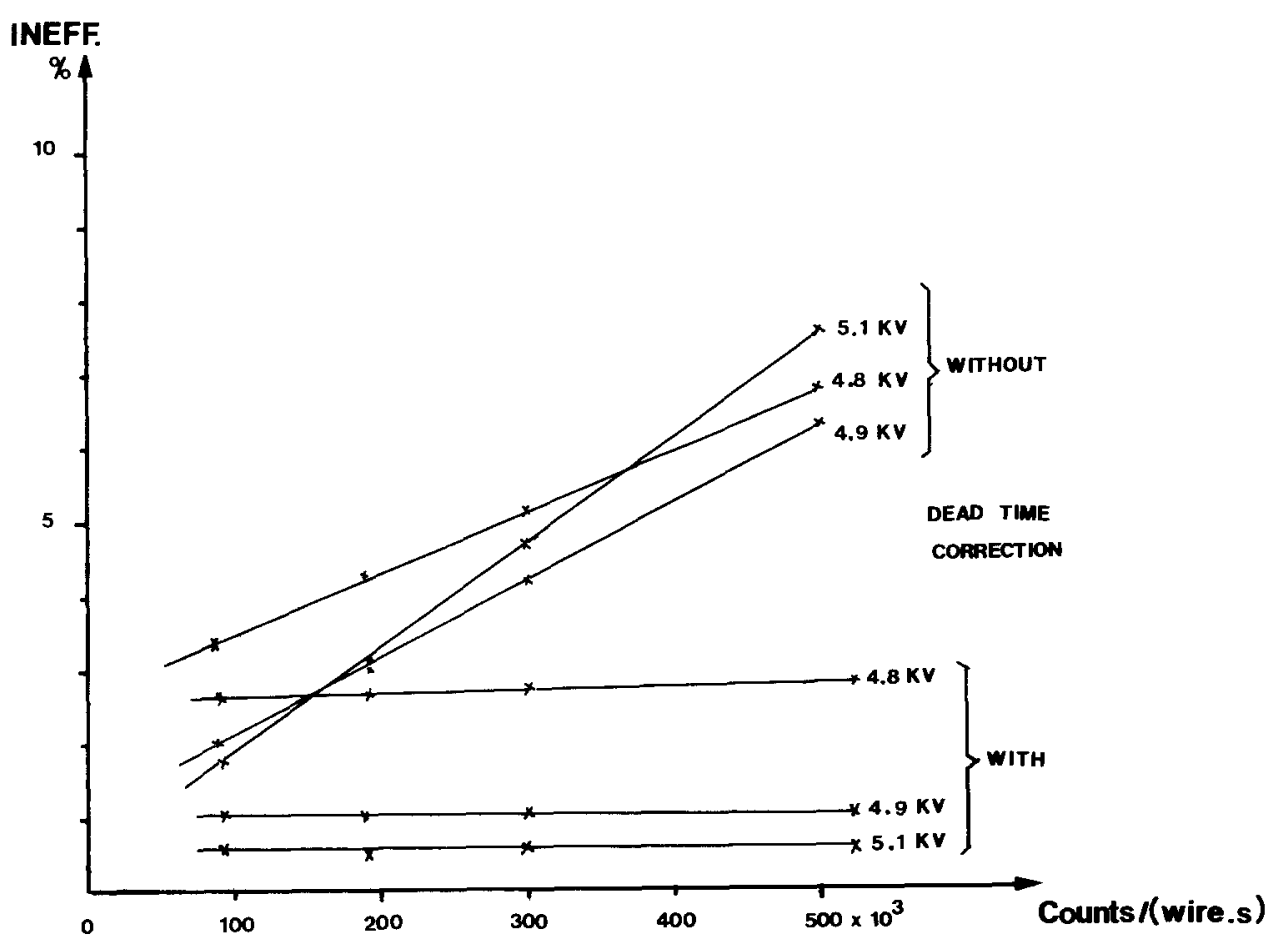

$5 \times 10^{5}$ particles/(wire.s) and for a double pulse resolution of $38 \mathrm{~ns}$, while in fig. 9 the inefficiency amounts to $7.5 \%$ and $6.2 \%$ at $5.1 \mathrm{kV}$ and $4.9 \mathrm{kV}$ respectively.

The importance of the space charge effect has been measured by scanning the scintillators along the wires, so as to change the beam density, keeping the four wires counting rate constant $( \pm 15 \%)$. In fig. 10 are reported the measured inefficiencies v.s. beam density for different working voltages (gains) and gas mixtures. The curves are compensated for the electronics dead time. The presence of Freon $(0.3 \%)$ improves slightly the chamber efficiency above a few $10^{4}$ particles $/\left(\mathrm{mm}^{2} \cdot \mathrm{s}\right)$.

The limitation of the chamber gain due to the positive ions barrier has been evaluated from the hv plateau at different densities for a constant rate/wire. The difference in voltage $(\Delta V)$ necessary to obtain the same efficiency $(85 \%)$ gives a figure of the reduction of the electric field around the wires due to the positive ions screening effect. With the $\mathrm{A}(50 \%)+\mathrm{CO}_{2}(50 \%)$ gas mixture the measured $\Delta V$ is about $200 \mathrm{~V}$ for $10^{5}$ particles $/\left(\mathrm{mm}^{2} \cdot \mathrm{s}\right)$, while the addition of $0.3 \%$ of Freon reduces $\Delta V$ to about $150 \mathrm{~V}$ for $10^{5}$ particles $/\left(\mathrm{mm}^{2} \cdot \mathrm{s}\right)$.

Fig. 9. Inefficiency curves vs counts/wire, at a density $\leq 5 \times 10^{3}$ particles $/\left(\mathrm{mm}^{2} \cdot \mathrm{s}\right)$, for different high voltages. The gas is $\mathrm{A}(50 \%)+\mathrm{CO}_{2}(50 \%)$ and the threshold $=0.5 \mu \mathrm{A}$. The importance of the occupation time is such that for a rate/(wire $\left.\cdot \mathrm{s}\right) \geq 2 \times 10^{5}$ the efficiency is maximum for high voltage $=4.9 \mathrm{kV}$. 

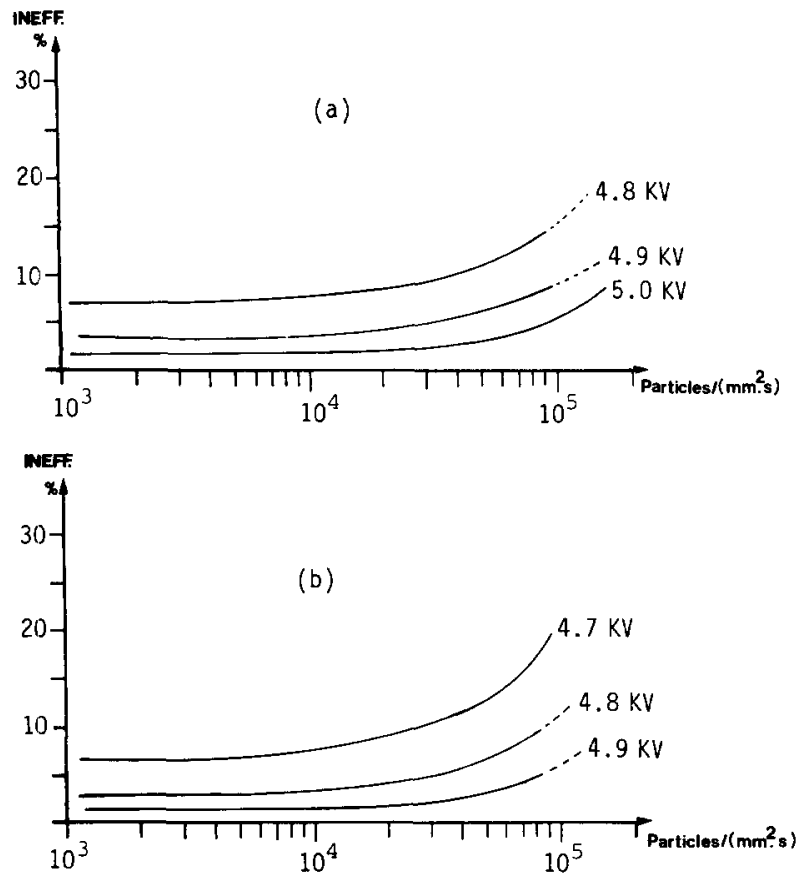

Fig. 10. Inefficiency curves vs beam density for different gains of the chamber with $\mathrm{A}(50 \%)+\mathrm{CO}_{2}(49.7 \%)+$ Freon $13 \mathrm{~B} 1(0.3 \%)$ (curve a) and $\mathrm{A}(50 \%)+\mathrm{CO}_{2}(50 \%)$ (curve b). The threshold is $0.5 \mu \mathrm{A}$ and the rate of the 4 wires $O R$ is about $2 \times 10^{6}$ counts $/ \mathrm{s}$. The curves are compensated for the electronics dead-time.

The real behaviour of the whole system (chamber and electronics) is represented in fig. 11, which shows an efficiency above $90 \%$ up to $10^{5}$ particles $/\left(\mathrm{mm}^{2} \cdot \mathrm{s}\right)$.

\section{Conclusions}

The results obtained in this test are very encouraging and largely above the initial aim, as far as the spectrometer's normal operation is concerned. Previous measurements ${ }^{4}$ ) with the same gas mixture show also a similar overall behaviour. Our measurements show that the electronics contribu-

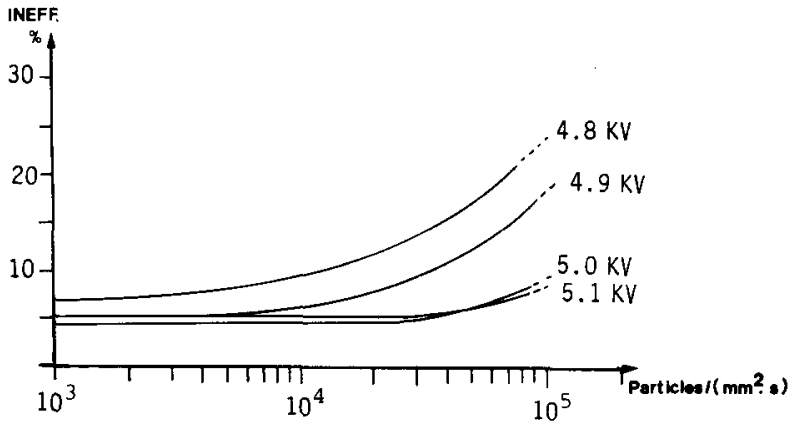

Fig. 11. Inefficiency curves vs beam density for different gains of the chamber without dead time corrections. The gas mixture is $\mathrm{A}(50 \%)+\mathrm{CO}_{2}(49.7 \%)+$ Freon $13 \mathrm{Bl}(0.3 \%)$ and the threshold $=0.5 \mu \mathrm{A}$.

tion to the inefficiency at high beam density and consequently at high rate/wire is of the same order of magnitude as the space charge effect.

The last phenomenon can be compensated by increasing the working voltage up to the value above which the contribution of the "occupation time" effect becomes predominant. Furthermore, an excessive high voltage, outside the region of maximum density, would make the all chamber operation quite unstable with an increasing risk of breakdown in peripheral regions.

We wish to thank P. Bouvier for his assistance in mounting the apparatus. We also acknowledge the efforts of Mr. M. Wenger in writing all the software for the measurements and Mr. D. E. Plane for the beam tuning.

\section{References}

1) G. Dubois, A. Placci, L. Pregernig, M. Rabany, B. Skaali and G. Vismara, Symp. on Nuclear science, San Francisco, U.S.A. (1977).

2) G. Vismara, to be published.

3) B. V. Cake, Technical Memorandum, Daresbury (1977).

4) P. Duerdoth, R. J. Ellison, R. E. H. Jones, D. Mercer, B. V. Cake and D. Clarke, Nucl. Instr. and Meth. 129 (1975) 461. 